\title{
Relevancia valorativa de información financiera y no financiera sobre capital intelectual en empresas cotizadas argentinas
}

\author{
Value relevance of financial and non-financial \\ information on intellectual capital in argentine \\ listed companies
}

\author{
Cecilia Ficco Pansarasa ${ }^{1}$, Gustavo Sader Bello ${ }^{2}$
}

\begin{abstract}
RESUMEN
El objetivo de este trabajo es analizar la relevancia que el mercado de capitales argentino asigna a la información financiera y no financiera referida al capital intelectual de las empresas que cotizan en el mismo. El análisis se realiza a través de un estudio correlacional en el que se utilizan modelos de precios basados en Ohlson (1995), los que se estiman utilizando la técnica de regresión lineal con datos de panel. Los datos se recolectan de fuentes originales y corresponden al período 2009-2015. Los resultados obtenidos muestran que solo los activos intangibles identificables y los datos de los estados financieros indicadores del capital humano y estructural de las empresas tienen relevancia para su valoración. En tanto que la información no financiera sobre capital intelectual, revelada voluntariamente, no es relevante para ninguna de las tres dimensiones del mismo. Estos hallazgos son acordes a los aportados por los estudios previos y, en particular, los referidos a la información no financiera de capital intelectual, son similares a los obtenidos para países de bajo desarrollo, donde no se han logrado evidencias sólidas que apoyen su relevancia valorativa.
\end{abstract}

Palabras clave: capital intelectual, información financiera, información no financiera, mercado de capitales argentino, relevancia valorativa.

Recepción: 26/08/2020, Aprobación: 11/11/2020.

1 Cecilia Ficco Pansarasa, Universidad Nacional de Río Cuarto, Facultad de Ciencias Económicas, Río Cuarto, Argentina, cficco@fce. unrc.edu.ar

2 Gustavo Sader Bello, Universidad Nacional de Río Cuarto, Facultad de Ciencias Económicas, Río Cuarto, Argentina, gusader@ hotmail.com 


\begin{abstract}
The objective of this work is to analyze the relevance that the argentine capital market assigns to financial and non-financial information referring to the intellectual capital of the companies listed on it. The analysis is carried out through a correlational study using price models based on Ohlson (1995), which are estimated using the linear regression technique with panel data. The data are collected from original sources and correspond to the period 2009-2015. The results obtained show that only the identifiable intangible assets and the data of the financial statements indicating the human and structural capital of the companies are relevant for their valuation. Non-financial information on intellectual capital, disclosed voluntarily, is not relevant for any of the three dimensions of the same. These findings are consistent with those provided by previous studies and, in particular, those referring to non-financial information on intellectual capital, are similar to those obtained for low-developed countries, where no solid evidence has been obtained to support its value relevance.
\end{abstract}

Key words: intellectual capital, financial information, non financial information, argentine capital market, value relevance.

\title{
INTRODUCCIÓN
}

El interés por los intangibles, o conjunto de elementos que conforman el capital intelectual (CI) de una organización, va incrementándose tras varias décadas de investigación desarrollada desde diferentes disciplinas. Una investigación que encuentra su plena justificación en un entorno como el actual en el que el conocimiento, sustrato fundamental en la creación de intangibles, parece presentarse como un recurso clave en la generación de riqueza organizacional.

En efecto, el CI refiere al conjunto de elementos intangibles (recursos, capacidades y actividades) relacionados entre sí, entre los que destaca el conocimiento disponible, tanto a nivel individual como organizativo, que permiten a la empresa funcionar, en combinación con los demás recursos inherentes a la misma, siendo fuente de ventaja competitiva y de creación de valor (Edvinsson y Malone, 1997; Stewart, 1998; Bueno, Salmador y Merino, 2008; Sardo y Serrasqueiro, 2018).

Se trata de un concepto holístico (Carlucci y Schiuma, 2007), que abarca todas las formas de elementos intangibles (Cañibano, Sánchez, García y Chaminade, 2002), las que la mayor parte de los autores coinciden en agrupar en tres grandes dimensiones: capital humano $(\mathrm{CH})$, capital estructural (CE) y capital relacional (CR).

El CH refiere al conocimiento tácito (Bontis, 1998) que reside en los empleados e incluye tanto los saberes que poseen los mismos, como sus capacidades, experiencias y habilidades (Cañibano et al., 2002). El CE es la infraestructura que incorpora, forma y sostiene al CH (Edvinsson y Malone, 1997), permitiendo optimizar su rendimiento (Bontis, 1998). Incluye rutinas organizativas, procedimientos y sistemas que permiten la conversión del conocimiento individual en colectivo (Bollen, Vergauwen y Schnieders, 2005), aunque involucra también los elementos que aluden 
a la capacidad de renovación de la empresa y los resultados de la innovación (Ochoa, Prieto y Santidrián, 2010), algunos de cuales "pueden protegerse legalmente y convertirse en derechos de propiedad intelectual o industrial, como los derechos de autor o las patentes" (Cañibano et al., 2002, p. 19). El CR refiere a la red de relaciones de la empresa con diferentes stakeholders (Ross, Ross, Dragonetti y Edvinson, 2001). Se trata pues de una dimensión externa, que incluye también los nombres de los productos, las marcas y la reputación o imagen de la empresa (Autora, 2020).

Es generalizado el reconocimiento del papel del CI como fuente fundamental de ventaja competitiva y de creación de valor. Sin embargo, es muy limitada la información sobre CI que se incorpora en los informes que se elaboran para atender a los requerimientos normativos vigentes. En este sentido, los organismos emisores de normas contables no han ofrecido una respuesta homogénea a esta problemática y, generalmente, son muy reducidos los activos intangibles que aparecen dentro de la información contable obligatoria que las empresas elaboran.

En este sentido, para que una inversión de naturaleza intangible pueda ser reconocida como activo, tanto la normativa contable de la Federación Argentina de Consejos Profesionales en Ciencias Económicas (FACPCE) como en las Normas Internacionales de Información Financiera (NIIF), emitidas por el International Accounting Standards Board (IASB), requieren la posibilidad de obtención de beneficios económicos futuros y la medición confiable, agregándose el requisito de identificabilidad en la NIC 38 del IASB (Autora, 2019). A su vez, estas normas distinguen dos categorías fundamentales de activos intangibles para regular su tratamiento, las que surgen de su clasificación por el criterio de identificabilidad, a saber: plusvalía y otros activos intangibles identificables (como patentes, marcas, licencias, franquicias, etc.). Con relación a la plusvalía, se admite solo el reconocimiento de la que fuera adquirida en una combinación de negocios.

Los requisitos antes expuestos imponen importantes restricciones para el reconocimiento de activos intangibles, por lo que muchos de los elementos que componen el CI de las empresas quedan fuera de sus estados financieros. Esta situación plantea importantes limitaciones a los sistemas contables en vigor para brindar información útil para que los inversores, que son considerados los principales usuarios a quienes se dirigen los informes financieros, puedan estimar adecuadamente el valor de las empresas y evaluar, así, los beneficios futuros y el riesgo asociados a las distintas oportunidades de inversión (Cañibano et al., 2002; Autora, 2020).

Frente a estas deficiencias informativas han surgido iniciativas tendientes a poner énfasis en la consideración de información no financiera, de tipo cualitativa/narrativa (Giner y Mora, 2019), la cual se encuentra estrechamente vinculada a elementos intangibles clave de la generación de valor público y organizacional, en tanto abarca un amplio abanico de temas sociales, medioambientales y otros relacionados con la sostenibilidad. Entre ellas se destacan los principios del Pacto Mundial de Naciones Unidas, los estándares de Global Reporting Initiative, las normas del Sustainability Accounting Standards Board y el marco del International Integrated Reporting Council.

Sin embargo, no se ha arribado a una solución de carácter general, por lo que, en el ámbito empresarial, la alternativa más utilizada para dar a conocer el CI es la de divulgar esta información de forma totalmente voluntaria, usando para ello diversos formatos y canales (información complementaria a los estados financieros, informes integrados o memorias de sostenibilidad). 
En Argentina, tanto los informes integrados como las memorias de sostenibilidad son de presentación voluntaria y son escasas las empresas que los elaboran. Por ello, a nivel general, la información referida al CI que las empresas revelan para su ámbito externo se encuentra disponible en los estados financieros y en la información que complementa a los mismos. En el primer caso, se trata de información que tiene carácter financiero, como es la referida a los intangibles que pueden reconocerse como activos y, así también, la referida a ciertos gastos que pueden considerarse como indicadores de inversiones en recursos y actividades intangibles. Dentro de la información complementaria, en cambio, se incluye, principalmente, información no financiera, de tipo cualitativo, la cual es incorporada en las notas a los estados financieros y en la memoria.

La situación problemática antes planteada ha contribuido al desarrollo de una importante línea de investigación tendiente a demostrar la "relevancia valorativa" de la información referida al CI, cuyo desarrollo resulta muy significativo en el marco de los cuestionamientos a la utilidad de la información contable, en tanto, tal como señala el IASB (2019), en su marco conceptual, la relevancia es una de las características cualitativas fundamentales de la información financiera útil.

La idea de "relevancia valorativa", o de relevancia para el valor, tiene diversas interpretaciones (Giner y Pardo, 2007). Sin embargo, una de las más usadas, es la que sostiene que la "relevancia valorativa" implica que una cifra contable (o no contable) está asociada con alguna medida del valor de la empresa, siendo el precio de las acciones la medida más comúnmente empleada por los investigadores (Barth, 2000). En este sentido, y tal como aclaran Barth, Beaver y Landsman (2001), una cifra contable (o no contable) es relevante para los inversores si posee una asociación significativa con los precios en el mercado de valores.

En este marco se plantea la presente investigación, la cual tiene como objetivo analizar la relevancia que el mercado de capitales asigna a la información financiera y no financiera referida al CI de las empresas que cotizan en el mismo. La investigación se realiza para el período temporal 2009-2015 y se contextualiza en el mercado de capitales argentino (MCA), donde no se han constatado trabajos publicados de esta naturaleza ${ }^{3}$.

La revisión de la literatura previa revela una abundante evidencia empírica sobre la relevancia valorativa del CI, aunque, en su mayoría, refiere a mercados de capitales desarrollados. En las investigaciones que han estudiado la asociación entre la información financiera sobre CI y los precios del mercado, se ha empleado como medida del mismo a la información referida a los activos intangibles reconocidos contablemente, junto a variables proxy basadas en medidas contables y destinadas a medir los distintos componentes que no tienen reflejo en los estados financieros, los que habitualmente se engloban en tres grandes bloques: $\mathrm{CH}, \mathrm{CE}$ y $\mathrm{CR}$. Los resultados de estos estudios varían de acuerdo a las distintas categorías analizadas. Así, en lo que respecta a los intangibles que tienen reconocimiento contable, existe sólidas evidencias en lo atinente a la relevancia valorativa de los activos intangibles identificables (Oliveira, Rodrigues y Craig, 2010; Kimouche y Rouabhi, 2016a; Da Silva, Rodrigues y Klann, 2017; Ocak y Findik, 2019), pero no resultan del todo concluyentes con relación a la plusvalía adquirida en las combinaciones de negocios, tal como surge de los trabajos de Kimouche y Rouabhi (2016b) e Infante y Ferrer (2017). Las eviden-

$3 \mathrm{El}$ presente trabajo forma parte de las investigaciones doctorales de sus autores y expone algunos resultados empíricos obtenidos a partir de las mismas. 
cias también son concluyentes en lo que respecta al impacto positivo del $\mathrm{CH}$ en la formación de precios, pero los resultados son menos consistentes en lo que respecta a la relevancia valorativa del CE y del CR (Wang, 2008; Liu, Tseng y Yen, 2009; Ferraro y Veltri, 2011; Nimtrakoom, 2015; Suherman, 2017; Sharma, 2018; Smriti y Das, 2018; Soetanto y Liem, 2019).

La literatura empírica que ha empleado información no financiera como subrogado del CI, en general, ha utilizado índices para su medición, los que son construidos por los propios investigadores para determinar la cantidad de información sobre CI que las empresas revelan voluntariamente. La evidencia aportada por estos trabajos no es concluyente. Así, Ferchichi y Paturel (2013) y Alfraih (2017) obtienen resultados que muestran que la divulgación del CI está asociada positiva y significativamente con el valor de mercado de las empresas. Sin embargo, otras investigaciones obtienen resultados que apoyan tal asociación, pero con limitaciones para ciertos sectores y componentes del CI (Whiting y Miller, 2008; Vafaei, Taylor y Ahmed, 2011).

Se aprecia, de este modo, que los trabajos empíricos previos reflejan resultados variados, siendo más consistentes para algunos componentes del CI. No obstante, la evidencia existente da sustento a la idea de que tanto la información financiera sobre CI, como la no financiera, puede tener efectos valorativos en el MCA. Particularmente, se espera que ambos tipos de información presenten una asociación positiva con el valor de mercado de las empresas, por ser información representativa de la creación de valor. Por ello, las hipótesis de la investigación se formulan de la siguiente manera:

H1: La información financiera referida al CI de las empresas cotizantes en el MCA tiene relevancia para la valoración de dichas empresas.

H2: La información no financiera referida al CI de las empresas cotizantes en el MCA tiene relevancia para la valoración de dichas empresas.

Tras esta introducción, el desarrollo del trabajo se ha estructurado en dos grandes secciones. En la primera se describe la metodología del estudio empírico realizado y, en la segunda, se presentan los resultados del mismo. Finamente se exponen las conclusiones y contribuciones que se derivan de tales resultados, su discusión y las perspectivas futuras de la investigación.

\section{METODOLOGÍA, MATERIALES Y MÉTODOS}

Construcción de modelos y definición de variables

Con el fin de atender al objetivo planteado en la introducción y contrastar las hipótesis formuladas, construimos dos modelos basados en Ohlson (1995), partiendo de la versión ampliamente usada por la investigación previa, que se especifica del siguiente modo:

$$
P_{i t}=\beta_{0}+\beta_{1} P N_{i t}+\beta_{2} R D O_{i t}+\beta_{3} v_{i t}+\varepsilon_{i t}
$$

donde $P_{i t}$ es el valor de mercado de las acciones de la empresa $i$ en el momento $t, P N_{i t}$ es el valor contable del patrimonio neto de la empresa $i$ en el momento $t, R D O_{i t}$ es el resultado contable 
de la empresa $i$ para el período $t$ y $v_{i t}$ es "otra información" que influye en el valor de la empresa $i$ pero que no está contenida en sus estados financieros en el momento $t$.

Para estudiar la relevancia valorativa de la información financiera referida al CI se modifica la expresión (1), en dos sentidos. Por un lado, se descompone $P N$ el en dos elementos: activos intangibles $(A I)$ y valor contable de los elementos tangibles netos (TGPN), siguiendo el planteo original de Ely y Waymire (1999). A su vez, los AI se consideran desglosados en sus dos categorías fundamentales: plusvalía $(P V)$ y otros activos intangibles identificables $(O A I)$. Por otro lado, se identifica a la variable "otra información" (v) con la referida a los elementos humanos $(\mathrm{CH})$, estructurales $(C E)$ y relacionales $(C R)$ de la empresa que no tienen reflejo en los estados financieros, pero que poseen una importante capacidad para producir efectos sobre su desempeño futuro (Wang, 2008; Liu et al., 2009; Ferraro y Veltri, 2011 y Veltri y Silvestri, 2011).

Para medir estos componentes se emplean proxies basadas en medidas contables. En la selección de las mismas se ha adoptado el enfoque basado en la inversión (Goebel, 2015; Forte, Tucker, Matonti y Nicolo, 2017), en tanto la mayoría de ellas se basan en gastos, relacionados al CI, que pueden ser considerados como inversiones con capacidad para generar beneficios económicos futuros vinculados al conocimiento existente dentro de la organización. Concretamente, se consideran dos proxies para cada dimensión, siguiendo la recomendación de Sveiby (1997), quien sugiere emplear pocos indicadores para mantener un sistema de medición simple y representativo. Así, para el $\mathrm{CH}$, se consideran los gastos de personal (GP), como una medida en términos absolutos de la inversión en dicho capital, junto a un indicador de productividad de tal inversión: la razón entre los ingresos por ventas y los gastos de personal (VTAS/GP), siguiendo el planteo de Veltri y Silvestri (2011), Nimtrakoom (2015), Smriti y Das (2018) y Sharma (2018). Los gastos administrativos y de comercialización $(G A y C)$ se emplean como una medida de la inversión en $C E$, y se utiliza también una medida relativa: la razón entre dichos gastos y los ingresos por ventas ( $G A y C$ ) VTAS), lo que se sustenta en los trabajos de Wang (2008), Ferraro y Veltri (2011) y Lev, Radhakrishnan, Evans (2016). El ingreso por ventas (VTAS) y el gasto en publicidad (PUB), se utilizan como variables proxy del $C R$, por ser las medidas consideradas como las más representativas de esta dimensión (Yu y Zhang, 2008; Ferraro y Veltri, 2011; Wang, 2008; Liu et al., 2009; Nimtrakoom, 2015; Suherman; 2017; Sharma, 2018).

De este modo, el modelo queda expresado tal como se muestra seguidamente:

$$
\begin{gathered}
P_{i t+3}=\beta_{0}+\beta_{1} T G P N_{i t}+\beta_{2} R D O_{i t}+\beta_{3} P V_{i t}+\beta_{4} O A I_{i t}+\beta_{5} G P_{i t}+ \\
\beta_{6} V T A S / G P_{i t}+\beta_{7} G A y C_{i t}+\beta_{8} G A y C / V T A S_{i t}+\beta_{9} V T A S_{i t}+\beta_{10} P U B_{i t}+\varepsilon_{i t}
\end{gathered}
$$

donde las variables se definen de acuerdo a lo indicado en el Cuadro 1.

Para estudiar la relevancia valorativa de la información no financiera referida al CI, también se identifica a la variable "otra información" (v) de (1) con la referida a los elementos humanos $(\mathrm{CH})$, estructurales $(C E)$ y relacionales $(C R)$ que no tienen reflejo en los estados financieros. Pero, en este caso, para medir cada una de esas dimensiones, se utilizan índices de revelación (IRCH, IRCE, $I R C R)$, que se construyen a partir de la información que las empresas divulgan voluntariamente a través de la memoria anual y de la información complementaria a los estados financieros. 
La utilización de índices de revelación responde a la aplicación del análisis de contenido (content analysis), que refiere a un conjunto de técnicas de investigación dirigidas al tratamiento de datos de tipo cualitativo generados en un proceso de comunicación (Autora y Autor, 2020). La aplicación de esta técnica para intentar medir la cantidad, calidad o cualquier otra característica de la información suministrada por las empresas implica enfrentarse con conceptos abstractos que no pueden ser medidos fácilmente de forma directa (Bernal y Reverte, 2016), por lo que, para la construcción de los índices de revelación (IRCH, IRCE, IRCR) se utiliza una metodología inductiva (Rodríguez, 2004) en la que se tienen en cuenta las particularidades del MCA para la selección de las categorías informativas referidas a cada dimensión del CI, las que quedaron definidas en veintisiete. Con relación a la codificación, se ha optado por el enfoque dicotómico y no ponderado, que es el mayoritariamente utilizado en los estudios previos (Giner, 1997; Larrán y Giner, 2002; García y Martínez, 2004; Patelli y Prencipe, 2007; Rodríguez, Gallego y García, 2010). El mismo implica utilizar valor cero y uno según se trate de ausencia o presencia de la categoría informativa ${ }^{4}$.

De esta manera, el modelo (3) queda expresado del siguiente modo:

$$
P_{i t+3}=\beta_{0}+\beta_{1} P N_{i t}+\beta_{2} R D O_{i t}+\beta_{3} I R C H_{i t}+\beta_{4} I R C E_{i t}+\beta_{5} I R C R_{i t}+\varepsilon_{i t}
$$

donde las variables se definen de acuerdo a lo indicado en el Cuadro 1.

Dos cuestiones merecen destacarse en lo relativo a la definición de las variables. En primer lugar, que la forma de cálculo de los precios de las acciones contempla los tiempos que demora el mercado en incorporar la información a las decisiones. En segundo lugar, los valores de las variables, que se obtienen de estados financieros de las empresas, se emplean deflactados por el número de acciones, a los efectos de mitigar los sesgos que produce el efecto escala en la estimación de modelos econométricos (Barth y Clinch, 2009).

La estructura de los datos disponibles, determinada por variables que se miden para cada empresa en distintos años, impone la necesidad de emplear modelos para datos correlacionados. Por ello, (2) y (3) se utilizan bajo la forma de modelos lineales para datos de panel, empleando una especificación de efectos fijos. Asimismo, para resolver los problemas de dispersión de los valores de la variable dependiente $(P)$, se trabaja con una transformación de la misma: el logaritmo natural del precio de la acción $(\operatorname{In} P)$.

De este modo, los modelos a estimar son los siguientes:

$$
\begin{gathered}
\ln P_{i t+3}=\beta_{0}+\beta_{1} T G P N_{i t}+\beta_{2} R D O_{i t}+\beta_{3} P V_{i t}+\beta_{4} O A I_{i t}+\beta_{5} G P_{i t}+ \\
\beta_{6} V T A S / G P_{i t}+\beta_{7} G A y C_{i t}+\beta_{8} G A y C / V T A S_{i t}+\beta_{9} V T A S_{i t}+\beta_{10} P U B_{i t}+\mu_{i}+\varepsilon_{i t} \\
\ln P_{i t+3}=\beta_{0}+\beta_{1} P N_{i t}+\beta_{2} R D O_{i t}+\beta_{3} I R C H_{i t}+\beta_{4} I R C E_{i t}+\beta_{5} I R C R_{i t}+\mu_{i}+\varepsilon_{i t}
\end{gathered}
$$

4 Los índices construidos se validan aplicando el índice Alpha de Cronbach, de acuerdo al cual valores mayores a 0,70 indican que existe coherencia interna. Los índices usados en este estudio presentan valores mayores al referido $(I R C H=0,9311 ; \operatorname{IRCE}=0,9253$; $I R C R=0,8945)$, por lo que se validan por su consistencia interna. 
donde $\mu_{i}$ es una variable aleatoria que permite captar la heterogeneidad individual no observable y las demás variables se definen de acuerdo a lo indicado en el Cuadro 1.

\section{Espacio temporal, muestra y fuentes de datos}

La investigación se realiza sobre una muestra de empresas cotizantes en el MCA pertenecientes a distintos sectores de actividad. El período elegido es el comprendido entre 2009 y 2015, ambos incluidos, lo que proporciona un rango temporal amplio para el análisis.

Dado que el MCA se caracteriza por una baja permanencia de las empresas en los índices bursátiles, para seleccionar a las que forman parte de la muestra se consideraron solo aquellas que se han mantenido en cotización de manera continua durante el período 2009-2015, identificándose 52 empresas en esta situación.

Para este conjunto se procedió a recolectar la información necesaria para la investigación, lo que permitió obtener un total de 364 observaciones anuales. De este total, se eliminaron las observaciones para las cuales las variables patrimonio neto y/o resultado contable asumen valores negativos, por no ser consistentes con el marco de valoración de Ohlson (1995). También se eliminaron valores atípicos e influyentes en los modelos de regresión, utilizando medidas estadísticas de uso habitual: residuos estudentizados, estadísticos DFFITS, COVRATIO y distancias de Cook y Welsch. En el proceso de detección de estos valores se trabajó, de manera independiente, con las variables explicativas de cada uno de los dos modelos construidos, quedando las muestras finales conformadas tal como se muestra en las Tablas 1 y 2.

Los datos se recolectaron de fuentes originales y se obtuvieron de los estados financieros anuales consolidados y de la memoria anual, presentados en la Bolsa de Comercio de Buenos Aires, y de los registros de negociación de acciones del Mercado de Valores de Buenos Aires. 


\section{Cuadro 1}

\section{Definición de variables}

\begin{tabular}{|c|c|}
\hline Simbología & Definición \\
\hline$P_{i t+3}$ & $\begin{array}{l}\text { Precio promedio por acción de la empresa } i \text { en el tercer mes siguiente al cierre del } \\
\text { ejercicio } t \text { (calculado tomando precios de cierres) }\end{array}$ \\
\hline$P N_{i t}$ & Patrimonio neto (por acción) de la empresa $i$ al cierre del ejercicio $t$ \\
\hline$T G P N_{i t}$ & Patrimonio neto tangible (por acción) de la empresa $i$ al cierre del ejercicio $t$ \\
\hline$R D O_{i t}$ & Resultado neto después de impuestos (por acción) de la empresa $i$ para el ejercicio $t$ \\
\hline$P V_{\text {it }}$ & $\begin{array}{l}\text { Plusvalía (por acción) informada en los estados financieros de la empresa } i \text { al cierre } \\
\text { del ejercicio } t \text { (neta de las depreciaciones }{ }^{5} \text { acumuladas y de las pérdidas por desvalo- } \\
\text { rización que le pudieran corresponder) }\end{array}$ \\
\hline$O A I_{i t}$ & $\begin{array}{l}\text { Otros activos intangibles (por acción) informados en los estados financieros de la } \\
\text { empresa } i \text { al cierre del ejercicio } t \text { (netos de las depreciaciones acumuladas y de las } \\
\text { pérdidas por desvalorización que les pudieran corresponder) }\end{array}$ \\
\hline$G P_{i t}$ & Gastos de personal (por acción) de la empresa $i$ para el ejercicio $t$ \\
\hline$V T A S / G P_{i t}$ & (Ventas / Gastos de personal) de la empresa $i$ para el ejercicio $t$ \\
\hline$G A y C_{i t}$ & $\begin{array}{l}\text { Gastos administrativos y de comercialización (por acción) de la empresa } i \text { para el } \\
\text { ejercicio } t \text { (netos de gastos de personal y de publicidad) }\end{array}$ \\
\hline GAyC/VTAS $i t$ & $\begin{array}{l}\text { (Gastos administrativos y de comercialización }{ }^{\star} / \text { Ventas) de la empresa } i \text { para el } \\
\text { ejercicio } t\left(^{\star} \text { netos de gastos de personal y de publicidad) }\right.\end{array}$ \\
\hline$V T A S_{i t}$ & Ingresos netos por ventas (por acción) de la empresa $i$ para el ejercicio $t$ \\
\hline$P U B_{i t}$ & Gastos de publicidad (por acción) de la empresa $i$ para el ejercicio $t$ \\
\hline $\mathrm{IRCH}_{i t}$ & Índice de revelación del capital humano de la empresa $i$ referido al ejercicio $t$ \\
\hline$I R C E_{i t}$ & Índice de revelación del capital estructural de la empresa $i$ referido al ejercicio $t$ \\
\hline$I R C R_{i t}$ & Índice de revelación del capital relacional de la empresa $i$ referido al ejercicio $t$ \\
\hline
\end{tabular}

Nota: El Cuadro 1 muestra la definición de las variables usadas en el estudio empírico. Fuente: elaboración propia.

5 Se emplea el término "depreciación", siguiendo la terminología usada por las normas contables argentinas. 


\section{RESULTADOS}

En esta sección se presentan los resultados obtenidos en la estimación de los modelos (4) y (5), los que se muestran en las Tablas 1 y 2 , respectivamente.

\section{Tabla 1}

Relevancia valorativa de la información financiera sobre CI

\begin{tabular}{ccc}
\hline Variables explicativas & Coeficientes & p-valores \\
\hline TGPN & $-0,0166$ & 0,716 \\
RDO & $0,2638^{* * *}$ & 0,008 \\
$P V$ & $-0,0701$ & 0,387 \\
OAI & $0,1901^{* *}$ & 0,013 \\
GP & $0,1545^{*}$ & 0,091 \\
VTAS/GP & $0,0047^{*}$ & 0,081 \\
GAyC & $0,1073^{* *}$ & 0,004 \\
GAyC/VTAS & $0,2495^{*}$ & 0,067 \\
VTAS & $-0,0080$ & 0,445 \\
PUB & $-0,5524$ & 0,234 \\
Constante & $1,1484^{* * *}$ & 0,000 \\
\hline $\mathrm{N}^{\circ}$ observaciones & 256 & \\
$\mathrm{~N}^{\circ}$ empresas & 46 & \\
$\mathrm{R}^{2}$ within & 0,4250 & \\
\hline Correlación intraclásica $($ rho $)$ & 0,7056 & \\
\hline
\end{tabular}

Nota: La Tabla 1 muestra los resultados de la estimación del modelo (4). Se ha utilizado el estimador dentro (within estimator) y se realizó estimación robusta a heterocedasticidad basada en la corrección de White (1980). Los símbolos ***, ** $\mathrm{y}$ * denotan que la variable es significativa al 1\%,5\% o 10\%, respectivamente. Las variables se definen de acuerdo a lo indicado en el Cuadro 1. Fuente: elaboración propia.

En lo que respecta a la información financiera sobre CI, los coeficientes y p-valores expuestos en la Tabla 1 muestran que las variables RDO, OAI, GP, VTAS/GP, GAyC/VTAS son estadísticamente significativas y positivas con un $90 \%$ de confianza, mientras que TGPN, PV, VTAS y PUB no lo son. Estos resultados indican que los activos intangibles identificables (OAI) proporcionan información relevante para los inversores, mientras que la plusvalía $(P V)$ no es significativa en el proceso de formación de precios. Respecto de los elementos del CI que no son reconocidos como activos, pero que tienen reflejo en los estados financieros a través de ciertos gastos e ingresos que pueden considerarse como proxies de los mismos, se evidencia que tanto el $\mathrm{CH}$ como el CE poseen relevancia valorativa, ya que las dos proxies del CH (GP, y VTAS/GP) y las dos proxies del CE (GAyC y GAyC/VTAS) son significativas y positivas. En cambio, ninguna de las proxies del CR (VTAS y PUB) son significativas, lo que pone de manifiesto que los elementos que componen esta dimensión no tienen impacto en el valor corporativo que asigna el mercado. 
De este modo, hemos obtenido evidencias que nos permiten aceptar parcialmente la hipótesis $\mathrm{H} 1$, ya que solo los activos intangibles identificables y la información financiera que es indicadora del $\mathrm{CH}$ y del CE de las empresas cotizantes en el MCA tiene relevancia para su valoración.

\section{Tabla 2}

\section{Relevancia valorativa de la información no financiera sobre CI}

\begin{tabular}{ccc}
\hline Variables explicativas & Coeficientes & p-valores \\
\hline$P N$ & 0,0603 & $0,015^{* *}$ \\
$R D O$ & 0,3030 & $0,000^{* * *}$ \\
$I R C H$ & $-0,1229$ & 0,600 \\
$I R C E$ & $-0,3010$ & 0,351 \\
IRCR & $-1,0510$ & 0,429 \\
Constante & 1.32813 & 0,000 \\
\hline $\mathrm{N}^{\circ}$ observaciones & 256 & \\
$\mathrm{~N}^{\circ}$ empresas & 47 & \\
$\mathrm{R}^{2}$ within & 0,3815 & \\
\hline Correlación intraclásica (rho $)$ & 0,7060 & \\
\hline
\end{tabular}

Nota: La Tabla 2 muestra los resultados de la estimación del modelo (5). Se ha utilizado el estimador dentro (within estimator) y se realizó estimación robusta a heterocedasticidad basada en la corrección de White (1980). Los símbolos *** y ** denotan que la variable es significativa al $1 \%$ o $5 \%$, respectivamente. Las variables se definen de acuerdo a lo indicado en el Cuadro 1. Fuente: elaboración propia.

En la Tabla 2 se aprecia que las variables clásicas del modelo de Ohlson (1995), PN y RDO resultaron significativas y con el signo esperado, por lo que influyen positivamente y son relevantes para el mercado de valores argentino.

Las variables IRCH, IRCE e IRCR no resultaron significativas, lo que evidencia que la información no financiera referida al $\mathrm{CH}, \mathrm{CE}$ y $\mathrm{CR}$ no es tomada en cuenta para la valoración de las empresas.

De este modo, los resultados obtenidos revelan que la información no financiera referida al CI de las empresas cotizantes en el MCA no tiene relevancia para su valoración por parte de los inversores, por lo que se rechaza la hipótesis H2.

\section{DISCUSIÓN Y CONCLUSIONES}

Los resultados obtenidos muestran que los inversores participantes en el MCA, para la toma de sus decisiones de compra y venta de acciones, tienen en cuenta la información sobre el CI de las empresas, pero solo la de carácter financiero.

La información no financiera sobre CI, de tipo cualitativa/narrativa, revelada voluntariamente por las empresas en forma complementaria a los estados financieros, no es relevante para los 
inversores en ninguna de sus tres dimensiones. Estos resultados son acordes a los obtenidos por estudios previos que han analizado el CI en mercados de capitales poco desarrollados, los que, en general, no han obtenido evidencias que apoyen su importancia para la toma de decisiones (Maditinos, Chatzoudes, Charalampos y Theriou, 2011; Sosa, 2013; Sharma, 2018). Esta falta de relevancia valorativa se podría fundamentar a partir de los hallazgos de Scott (2009) sobre información voluntaria relacionada con planes y expectativas futuras, quien aporta evidencia contradictoria en relación con la utilidad de la misma para los inversores, atribuyéndolo, entre otras razones, a las dificultades que se presentan, a nivel del mercado, para estimar la información proveniente de fuentes distintas a los estados financieros y al hecho de que la información puede ser pertinente pero no confiable, en virtud de las estimaciones y supuestos que se hacen para su preparación.

Asimismo, las evidencias obtenidas revelan que solo una parte de la información sobre CI, contenida en los estados financieros, tiene relevancia para la valoración de las empresas; específicamente, la referida a los activos intangibles identificables y a los elementos que componen el $\mathrm{CH}$ y el CE. Estos resultados sugieren que, a pesar de los problemas que plantea el reconocimiento y la medición de los activos intangibles, los inversores perciben a los identificables como activos legítimos, considerando que proporcionan información sobre los futuros beneficios de las empresas y que contribuyen a los mismos. La plusvalía, en cambio, no resultó una variable de interés para los inversores, lo puede entenderse vinculado al hecho de que esta cifra contable -tal y como señalan Giner y Pardo (2007) - no expresa el valor del intangible, excepto en el momento de la combinación de empresas. Los resultados también revelan que el MCA comprende la importancia del $\mathrm{CH}$, como componente primario y clave del CI, y así también, la trascendencia del CE como sostén y elemento potenciador del primero. No obstante, el CR no resultó significativo, lo que es compresible si se tiene en cuenta, como indica Sveiby (1997), que se trata de una dimensión externa, que es afectada también por factores ajenos a las empresas.

Los elementos intangibles que resultan relevantes para los inversores participantes en el MCA son coincidentes con aquellos sobre los cuales la literatura previa ha aportado las evidencias más sólidas. Así, nuestros hallazgos reconfirman los de Oliveira et al. (2010), Kimouche y Rouabhi (2016a y b), Da Silva et al. (2017) y Ocak y Findik (2019), que comprueban que los inversores tienen en cuenta a los activos intangibles identificables en la fijación del precio de las acciones, y los de Wang (2008), Liu et al. (2009), Nimtrakoom (2015) y Suherman (2017), que encuentran evidencias que apoyan la relevancia valorativa tanto del $\mathrm{CH}$ como del CE. Nuestros resultados también están en sintonía con los estudios previos que han analizado la relevancia valorativa de la plusvalía y del CR, los que no han logrado aportar evidencia contundente al respecto, tal como se refleja en los trabajos de Yu y Zhang (2008), Wang (2008), Kimouche y Rouabhi (2016b), Infante y Ferrer (2017) y Soetanto y Liem (2019).

Los hallazgos que derivan de los resultados obtenidos contribuyen a acrecentar la evidencia empírica existente en torno al papel del CI en la valoración externa de las empresas, la cual es necesaria para dar sustento a la construcción de una teoría, aún no desarrollada, que permita explicar la influencia de los intangibles en la creación de valor. Además, la investigación aporta al conocimiento de la forma en que la información referida al CI impacta en los mercados poco desarrollados, para los cuales la evidencia empírica existente es más limitada. Este trabajo también tiene potencial para contribuir a la regulación contable, en la medida que los resultados contribu- 
yen a hacer más patente la necesidad de incluir una mayor cantidad de información referida al $\mathrm{CI}$ en los estados financieros, o en estados complementarios, en pos de incrementar su utilidad.

Para dar continuidad a la línea de investigación aquí planteada, se proponen distintas alternativas. Por un lado, resultaría valioso ampliar la muestra a los fines de incluir empresas de otros países latinoamericanos, con el objeto de indagar acerca de los efectos de las características culturales e institucionales en la relevancia valorativa del CI. Por otro lado, resultaría de interés analizar el efecto que puede tener el tamaño de las empresas y su sector en la relevancia valorativa del CI, lo que aportaría a una comprensión más acabada de la relación entre intangibles y generación de valor.

\section{REFERENCIAS BIBLIOGRAFICAS}

Alfraih, M. (2017). The value relevance of intellectual capital disclosure: empirical evidence from Kuwait. Journal of Financial Regulation and Compliance, 25(1), 22-38.

Barth, M., Beaver, W. y Landsman, W. (2000). The relevance of the value relevance research. Working Paper, Stanford University, USA.

Barth, M., Beaver, W. y Landsman, W. (2001). The relevance of the value relevance literature for financial accounting standard setting: another view. Journal of Accounting and Economics, 31(1-3), 77-104.

Barth, M. y Clinch, G. (2009). Scale effects in capital markets-based accounting research. Journal of Business Finance \& Accounting, 36(3-4), 253-288.

Bernal, J. y Reverte, C. (2016). Herramienta de valoración de empresas. Estrategia Financiera. 336, 8-19.

Bollen, L., Vergauwen, P. y Schnieders, S. (2005). Linking intellectual capital and intellectual property to company performance. Management Decision, 43(9), 1161-1185.

Bontis, N. (1998). Intellectual capital: An exploratory study that develops measures and models. Management Decision, 36(2), 63-76.

Bueno, E., Salmador, M. y Merino, C. (2008). Génesis, concepto y desarrollo del capital intelectual en la economía del conocimiento: Una reflexión sobre el Modelo Intellectus y sus aplicaciones. Estudios de Economía Aplicada, 26(2), 43-63.

Cañibano, L., Sánchez, M. García, M. y Chaminade, C. (2002). Directrices para la gestión y difusión de información sobre intangibles (Informe de Capital Intelectual). Proyecto Meritum. Madrid: Fundación Airtel Móvil.

Carlucci, D. y Schiuma, G. (2007). Exploring intellectual capital concept in strategic management research. En L. Joia (Ed.), Strategies for Information Technology and Intellectual Capital: Challenges and Opportunities (pp. 10-28). London: Idea Group.

Da Silva, A., Rodrigues, T. y Klann, R. (2017). A influência dos ativos intangíveis na relevância da informação contábil. Revista Contemporânea de Contabilidade, 14(31), 26-45.

Edvinsson, L. y Malone, M. (1997). Intellectual Capital. Realizing your company's true value by findings its hidden brainpower. New York: Harper Collins Publishers.

Ely, K. y Waymire, G. (1999). Accounting standard-setting organizations and earnings relevance: Longitudinal evidence from NYSE common stocks, 1927-93. Journal of Accounting Research, 37(2), 293-317. 
Federación Argentina de Consejos Profesionales de Ciencias Económicas (2020). Resoluciones Técnicas vigentes. Buenos Aires: FACPCE.

Ferchichi, J. y Paturel, R. (2013). The effect of intellectual capital disclosure on the value creation: An empirical study using Tunisian annual reports. International Journal of Accounting and Financial Reporting, 3(1), 81-107.

Ferraro, O. y Veltri, S. (2011). The value relevance of intellectual capital on the firm's market value: An empirical survey on the Italian listed firms. International Journal of Knowledge-Based Development, 2(1), 66-84.

Autora (2019). Los activos intangibles en la normativa contable argentina y en las normas internacionales de información financiera. Contabilidad y Auditoría, 50, 61-108.

Autora (2020). Relevancia valorativa de los activos intangibles y del capital intelectual: Una revisión de la literatura empírica. Revista Academia \& Negocios, 6(1).

Autora y Autor (2019). Relevancia valorativa de los intangibles: Análisis pre-NIIF y NIIF en el mercado de capitales argentino. CAPIC Review, 17, 1-15.

Forte, W., Tucker, J., Matonti, G. y Nicolo, G. (2017). Measuring the intellectual capital of Italian listed companies. Journal of Intellectual Capital, 18(4), 710-732.

García, E. y Martínez, I. (2004). Divulgación voluntaria de información empresarial: índices de revelación. Partida Doble, 157, 66-77.

Giner, B. (1997). The influence of company characteristic and accounting regulation on information disclosed by Spanish firms. The European Accounting Review, 6(1), 45-68.

Giner, B. y Mora, A. (2019). A vueltas con los objetivos de la contabilidad: ¿un nuevo paradigma contable? Revista AECA, 127, 39-42.

Giner, B. y Pardo, F. (2007). La relevancia del fondo de comercio y su amortización en el mercado de capitales: Una perspectiva europea. Revista Española de Financiación y Contabilidad, 134(36), 389-415.

Goebel, V. (2015). Estimating a measure of intellectual capital value to test its determinants. Journal of Intellectual Capital, 16(1), 101-120.

Infante, R. y Ferrer, R. (2017). The impact of intangibles on the value relevance of accounting information: An empirical examination of listed companies in the Philippines from 2012 to 2016. Proceedings of the Institute for Global Business Research Conference, USA.

International Accounting Standards Board (2019). Normas Internacionales de Información Financiera vigentes. London: IASB.

Kimouche, B. y Rouabhi, A. (2016a). The impact of intangibles on the value relevance of accounting information: Evidence from French companies. Intangible Capital, 12(2), 506-529.

Kimouche, B. y Rouabhi, A. (2016b). Intangibles and value relevance of accounting information: Evidence from UK companies. Jordan Journal of Business Administration, 12(2), 437-458.

Larrán, M. y Giner, B. (2002). The use of the internet for corporate reporting by Spanish companies. The International Journal of Digital Accounting Research, 2(1), 53-82.

Lev, B., Radhakrishnan, S. y Evans, P. (2016). Organizational capital. A CEO's guide to measuring and managing enterprise intangibles. New York: Center for Global Enterprise.

Liu, D., Tseng, K. y Yen, S. (2009). The incremental impact of intellectual capital on value creation. Journal of Intellectual Capital, 10(2), 260-276.

Maditinos, D., Chatzoudes, D., Charalampos, T. y Theriou, G. (2011). The impact of intellectual capital on firms' market value and financial performance. Journal of Intellectual Capital, 12(1), 132-151. 
Nimtrakoon, S. (2015). The relationship between intellectual capital, firms' market value and financial performance: Empirical evidence from the ASEAN. Journal of Intellectual Capital, 16(3), 587-618.

Ochoa, M., Prieto, B. y Santidrián, A. (2010). Estado actual de los modelos de capital intelectual y su impacto en la creación de valor en empresas de Castilla y León. Revista de Investigación Económica y Social de Castilla y León, 13, 15-205.

Ohlson, J. (1995). Earnings, book values and dividends in equity valuation. Contemporany Accounting Research, 11(2), 661-687.

Ocak, M. y Findik, D. (2019). The impact of intangible assets and sub-components of intangible assets on sustainable growth and firm value: Evidence from Turkish listed firms. Sustainability, 11(19), 5359.

Oliveira, L., Rodrigues, L. y Craig, R. (2010). Intangible assets and value relevance: Evidence from the Portuguese stock exchange. The British Accounting Review, 42(4), 241-252.

Patelli, L. y Prencipe, A. (2007). The relationship between voluntary disclosure and independent directors in presence of a dominant shareholder. European Accounting Review, 16(1), 5-33.

Rodríguez, G. (2004). Factores explicativos de la revelación voluntaria de información. Revista Española de Financiación y Contabilidad, 33(122), 705-739.

Rodríguez, L., Gallego, I. y García, I. (2010). Determinantes de la divulgación voluntaria de información estratégica en internet: Un estudio de las empresas españolas cotizadas. Revista Europea de Dirección y Economía de la Empresa, 19(1), 9-26.

Roos, G., Roos, J., Dragonetti, N. y Edvinsson, L. (2001). Capital Intelectual. Bs. Aires: Paidós.

Sardo, F. y Serrasqueiro, Z. (2018). Intellectual capital, growth opportunities, and financial performance in European firms: Dynamic panel data analysis. Journal of Intellectual Capital, 19(4), 747-767.

Scott, W. (2009). Financial Accounting Theory. Toronto: Pearson Prentice Hall

Sharma, P. (2018). Enterprise value and intellectual capital: Study of BSE 500 firms. Accounting and Finance Research, 7(2), 123-133.

Smriti, N. y Das, N. (2018). The impact of intellectual capital on firm performance: A study of Indian firms listed in COSPI. Journal of Intellectual Capital, 19(5), 935-964.

Soetanto, T. y Liem, P. (2019). Intellectual capital in Indonesia: Dynamic panel approach. Journal of Asia Business Studies, 13(2), 240-262.

Sosa, M. (2013). El capital intelectual en mercados en desarrollo. El caso de Paraguay. Tesis Doctoral, Universidad de Sevilla, España.

Stewart, T. (1998). La nueva riqueza de las organizaciones: El capital intelectual. Buenos Aires: Ediciones Granica.

Sveiby, K. (1997). The intangible assets monitor. Recuperado el 8 de marzo de 2019, desde: https: / / www.sveiby.com/article/The-Intangible-Assets-Monitor

Suherman, R. (2017). The impact of intellectual capital toward firm's profitability and market value of retail companies listed in Indonesia Stock Exchange (IDX) from 2013-2016. iBuss Management, 5(1), 98-112.

Vafaei, A., Taylor, D. y Ahmed, K. (2011). The value relevance of intellectual capital disclosures. Journal of Intellectual Capital, 12(3), 407-429.

Veltri, S. y Silvestri, A. (2011). Direct and indirect effects of human capital on firm value: Evidence from Italian companies. Journal of Human Resource Costing \& Accounting, 15(3), 232-254.

Wang, J. (2008). Investigating market value and intellectual capital for SyP 500. Journal of Intellectual Capital, 9(4), 546-563. 
White, H. (1980). A heteroskedasticity-consistent covariance matrix estimator and a direct test for heteroskedasticity. Econometrica, 48(4), 817-838.

Whiting, R. y Miller, J. (2008). Voluntary disclosure of intellectual capital in New Zealand annual reports and the "hidden value". Journal of Human Resource Costing \& Accounting, 12(1), 2650.

Yu, F. y Zhang, L. (2008). Does intellectual capital really create value? $4^{\text {th }}$ International Conference on Wireless Communications, Networking and Mobile Computing, China.

\section{(c)}

Esta obra está bajo una licencia de Creative Commons Atribución-NoComercial-CompartirIgual 4.0 Internacional 